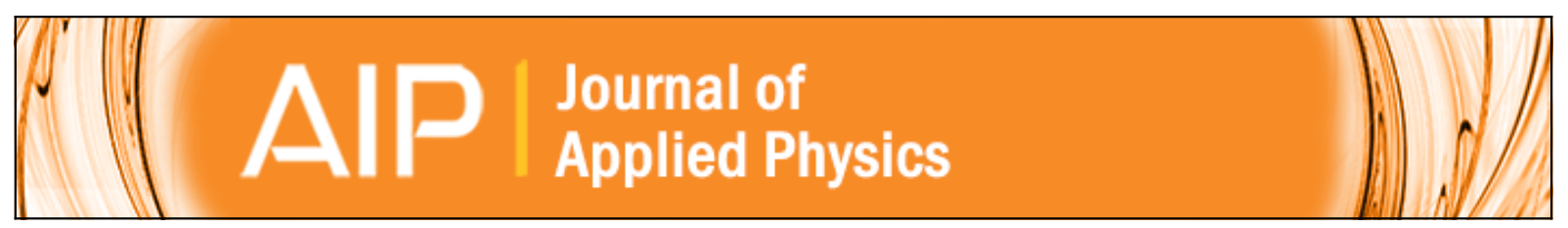

Characterization and modeling of a $\mathrm{ZnO}$ nanowire ultraviolet photodetector with graphene transparent contact

H. Zhang, A. V. Babichev, G. Jacopin, P. Lavenus, F. H. Julien, A. Yu. Egorov, J. Zhang, T. Pauporté, and M. Tchernycheva

Citation: Journal of Applied Physics 114, 234505 (2013); doi: 10.1063/1.4854455

View online: http://dx.doi.org/10.1063/1.4854455

View Table of Contents: http://scitation.aip.org/content/aip/journal/jap/114/23?ver=pdfcov

Published by the AIP Publishing

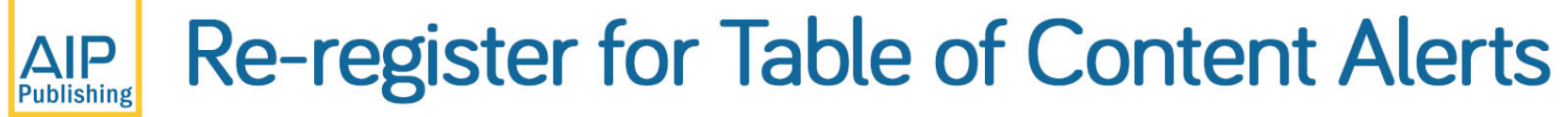




\title{
Characterization and modeling of a $\mathrm{ZnO}$ nanowire ultraviolet photodetector with graphene transparent contact
}

\author{
H. Zhang, ${ }^{1}$ A. V. Babichev, ${ }^{1,2,3}$ G. Jacopin, ${ }^{1,4}$ P. Lavenus, ${ }^{1}$ F. H. Julien, ${ }^{1}$ A. Yu. Egorov,${ }^{2}$ \\ J. Zhang, ${ }^{5}$ T. Pauporté, ${ }^{5}$ and M. Tchernycheva ${ }^{1, a)}$ \\ ${ }^{1}$ Institut d'Electronique Fondamentale, UMR 8622 CNRS, University Paris Sud XI, 91405 Orsay cedex, \\ France \\ ${ }^{2}$ St. Petersburg Academic University, Nanotechnology Research and Education Centre, \\ Russian Academy of Science, Khlopina 8/3, 194021 St. Petersburg, Russia \\ ${ }^{3}$ Ioffe Physical-Technical Institute of the Russian Academy of Science, Polytechnicheskaya 26, \\ 194021 St. Petersburg, Russia \\ ${ }^{4}$ ICMP LOEQ Ecole Polytechnique Fédérale de Lausanne, 1015 Lausanne, Switzerland \\ ${ }^{5}$ Laboratoire d'Electrochimie, Chimie des Interfaces et Modélisation pour l'Energie, UMR-CNRS 7575 , \\ Ecole Nationale Supérieure de Chimie de Paris, 11 rue P. et M. Curie, 75231 Paris cedex 05, France
}

(Received 28 October 2013; accepted 9 December 2013; published online 20 December 2013)

\begin{abstract}
We report the demonstration of a $\mathrm{ZnO}$ nanowire ultraviolet photodetector with a top transparent electrode made of a few-layered graphene sheet. The nanowires have been synthesized using a lowcost electrodeposition method. The detector is shown to be visible-blind and to present a responsivity larger than $10^{4} \mathrm{~A} / \mathrm{W}$ in the near ultraviolet range thanks to a high photoconductive gain in $\mathrm{ZnO}$ nanowires. The device exhibits a peak responsivity at $370 \mathrm{~nm}$ wavelength and shows a sub bandgap response down to $415 \mathrm{~nm}$ explained by an Urbach tail with a characteristic energy of $83 \mathrm{meV}$. The temporal response of the detector and the power dependence are discussed. A model of the photoconductive mechanism is proposed showing that the main process responsible for the photoconductive gain is the modulation of the conducting surface due to the variation of the surface depletion layer and not the reduction of recombination efficiency stemming from the electron-hole spatial separation. The gain is predicted to decrease at high incident power due to the flattening of the lateral band bending in agreement with experimental data. (C) 2013 AIP Publishing LLC. [http://dx.doi.org/10.1063/1.4854455]
\end{abstract}

\section{INTRODUCTION}

Near ultra violet (UV) light detection is important for many applications such as environmental monitoring, flame detection, military applications, and industrial quality control. ${ }^{1-4}$ Contrary to Si-based devices, which are widely used for these applications, $\mathrm{ZnO}$-based detectors have the advantage to be insensitive to the visible light allowing for visible-blind detection without any additional filters. ${ }^{5}$ The interest in using $\mathrm{ZnO}$ nanowires for UV detection has emerged in the early years 2000 with the demonstration of highly sensitive photodetectors based on individual nanowires. ${ }^{6}$ One particular advantage of $\mathrm{ZnO}$ nanowires is that it is a low-cost material that can be solution-synthesized on a vast variety of substrates. ${ }^{7-9}$ A giant photoconductive gain of $10^{8}$ leading to very high detector responsivity has been reported in single nanowire $\mathrm{ZnO}$ photodetectors and attributed to hole trapping at the nanowire surface due to oxygen chemisorption. ${ }^{10} \mathrm{It}$ has been demonstrated that the nanowire surface passivation allows to reach high detectivity values, while maintaining high responsivity of the detector. ${ }^{11}$ An extensive literature review on $\mathrm{ZnO}$ nanowire photodetectors can be found in Ref. 9

Recently, $\mathrm{ZnO} /$ carbon hybrid nanomaterials have attracted a great attention as a way to further improve the $\mathrm{ZnO}$

\footnotetext{
a) Author to whom correspondence should be addressed. Electronic mail: maria.tchernycheva@u-psud.fr
}

nanowire detector performance. The motivation is to take advantage of the strong UV response of $\mathrm{ZnO}$ nanowires combined with the excellent transport properties of transparent and low-cost carbon-based electrodes. Ultraviolet photodetectors based on single $\mathrm{ZnO}$ nanowires sandwiched between two graphene sheets on a $\mathrm{Si} / \mathrm{SiO}_{2}$ substrate have been demonstrated. ${ }^{12}$ Single-walled carbon nanotube electrodes have been employed to fabricate a photodetector based on $\mathrm{ZnO}$ nanowire ensembles. ${ }^{13}$ Finally, ZnO/graphene composite has been used as UV sensitive material, in which the graphene obtained by chemical reduction of graphene oxide plays the role of a charge transporting highly conductive matrix and the $\mathrm{ZnO}$ nanowires enhance the light absorption. ${ }^{14} \mathrm{~A}$ suspended graphene contact on $\mathrm{ZnO}$ nanowires has been used to fabricate flexible and transparent gas sensors. ${ }^{15} \mathrm{ZnO}$ nanowire ensemble/graphene photodetector based on a Schottky diode operation principle has been reported recently with a responsivity around $100 \mathrm{~A} / \mathrm{W}^{16} \mathrm{~A}$ higher responsivity can be achieved with a photoconductive device by taking advantage of a high photoconductive gain in nanowires.

In this work, we report the fabrication and characterization of a photoconductive detector based on $\mathrm{ZnO}$ nanowire ensembles with a transparent graphene top electrode. The $\mathrm{ZnO}$ nanowires are synthesized by the electrodeposition method on F-doped $\mathrm{SnO}_{2}$ (FTO) glass substrate. ${ }^{17,18}$ The CVD-grown graphene layers are transferred from $\mathrm{Ni} / \mathrm{SiO}_{2} / \mathrm{Si}$ substrate to the nanowire tops by a wet transfer method and then metal contacts are defined by optical lithography. The 
fabricated device presents a strong response to near-UV illumination with a responsivity larger than $10^{4} \mathrm{~A} / \mathrm{W}$ at $1 \mathrm{~V}$ bias. The detector response is peaked at $370 \mathrm{~nm}$. A sub-bandgap photoresponse is present up to $\lambda=415 \mathrm{~nm}$ attributed to the Urbach tail with a characteristic energy of $83 \mathrm{meV}$. The photocurrent exhibits multi-exponential time dependence with characteristic times for the current decay (25s and 254s) that is longer than for the current build-up (8s and 142s). The photocurrent dependence on the incident power is shown to be linear at low excitation, it becomes sub-linear for power densities above $10^{-4} \mathrm{~W} / \mathrm{cm}^{2}$ and it almost saturates above $10^{-2} \mathrm{~W} / \mathrm{cm}^{2}$. A model of the photoconductive mechanism is proposed, which unlike the widely adopted model of Soci et al., ${ }^{10}$ explicitly takes into account the effect of the modulation of the conducting surface due to the variation of the surface depletion layer. It is shown that this effect has a predominant contribution to the photoconductive gain compared to the slowing down of the electron-hole recombination due to the spatial carrier separation. The proposed model well describes the power dependence of the photoconductive gain and provides quantitative agreement with the experimental data.

\section{NANOWIRE SYNTHESIS AND CHARACTERIZATION}

The $\mathrm{ZnO}$ nanowire arrays were grown using an electrochemical deposition (ECD) technique. ${ }^{19,20}$ The deposition bath contained $0.2 \mathrm{mM} \mathrm{ZnCl} 2$ (Merck, 99\%) and $0.1 \mathrm{M}$ of potassium chloride $(\mathrm{KCl})$ (Fluka 99\%) was employed to ensure a good electrical conductivity in the aqueous solution (Milli-Q quality water). The $\mathrm{pH}$ of the solutions was 5.5 initially and it was bubbled with molecular oxygen. The three-electrode electrochemical cell was mounted in a thermoregulated bath, and the temperature was fixed at $85^{\circ} \mathrm{C}$ with an uncertainty of $\pm 0.2^{\circ} \mathrm{C} .{ }^{21}$ The $\mathrm{ZnO}$ nanowires were grown on a conductive FTO layer deposited on a glass substrate with a sheet resistance of $10 \Omega /$ sq. A constant $-1.0 \mathrm{~V} / \mathrm{SCE}$ potential was applied during $2700 \mathrm{~s}$ using an Autolab PGSTAT30 potentiostat/galvanostat. The working electrode was rotated with constant speed of $\omega=300$ rotations/min (rpm) to insure the high homogeneity of the deposited layer. ${ }^{22}$ After growth, the nanowire ensembles were thoroughly rinsed with milliQ water, dry in air and were subsequently annealed at $400{ }^{\circ} \mathrm{C}$ for $1 \mathrm{~h}$ to reduce the residual n-type doping.

The nanowire morphology was first characterized by scanning electronic microscopy (SEM) as illustrated in the inset to Fig. 1. The growth process results in a dense nanowire ensemble (density is approx. 10 nanowires per $\mu \mathrm{m}^{2}$ ) with a predominant vertical orientation. The average nanowire diameter was $150 \mathrm{~nm}$ and the nanowire length was about $1 \mu \mathrm{m}$.

The optical properties of $\mathrm{ZnO}$ nanowires were probed by photoluminescence (PL) spectroscopy. PL characterization was carried out at room temperature by exciting the sample with a continuous wave $244 \mathrm{~nm}$ light from a frequency-doubled $\mathrm{Ar}^{++}$ion laser. The luminescence was dispersed with a $460 \mathrm{~mm}$ focal length spectrometer and detected with a charge coupled device (CCD) camera. A

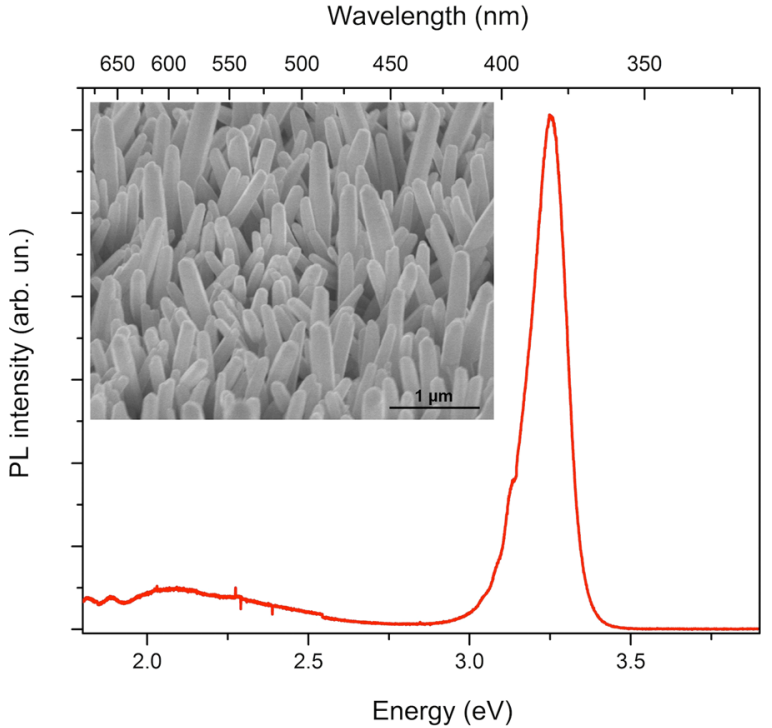

FIG. 1. Room temperature PL spectrum of $\mathrm{ZnO}$ nanowires. Inset $-45^{\circ}$-tilted SEM image of the as-grown nanowires.

typical spectrum of the nanowire ensemble is shown in Figure 1. The PL spectrum shows a strong near-band-edge (NBE) emission peak centered at $\sim 3.25 \mathrm{eV}(380 \mathrm{~nm})$ with a full width at half maximum of $130 \mathrm{meV}$. A weak yellow-green luminescence is observed around $600 \mathrm{~nm}$, which is related to defects and oxygen vacancies in $\mathrm{ZnO}$ nanowires. ${ }^{23}$ The defect emission is more than ten times weaker than the NBE peak, which demonstrates high material quality of the solution-synthesized $\mathrm{ZnO}$ nanowires in good agreement with previous studies. ${ }^{7,23}$

\section{DEVICE FABRICATION}

The nanowires were encapsulated into spin-on glass (hydrogen silsesquioxane), which were transformed into $\mathrm{SiOx}$ by $1 \mathrm{~h}$ thermal annealing at $400{ }^{\circ} \mathrm{C}$ under $\mathrm{N}_{2}$ flux. The excess of spin-on glass was etched using reactive ion etching with $\mathrm{CF}_{4}$ chemistry to uncover the nanowire tips (Fig. 2(a)). Commercially available approximately 4-monolayer thick graphene was used for the top transparent electrode. The graphene is synthesized by chemical vapor deposition on $\mathrm{Si} / \mathrm{SiO}_{2} / \mathrm{Ni}$ substrates (details of growth can be found in Ref. 24). The graphene sheet was separated from its growth substrate by first covering the surface with a $50 \mathrm{~nm}$ thick PMMA layer and then by etching the $\mathrm{Ni}$ underlayer using $\mathrm{FeCl}_{3}$ wet attack. The separated floating graphene sheet was transferred to the top surface of $\mathrm{ZnO}$ nanowire ensemble with the wet transfer method as described in Ref. 25. The size of the transferred graphene sheet is approx. $5 \times 5 \mathrm{~mm}^{2}$. After naturally drying the sample, the surface PMMA layer was removed by acetone and the polymer remainings were burned by annealing the sample at $300^{\circ} \mathrm{C}$ in nitrogen atmosphere (Fig. 2(b)). The metal contact with an open central part was then defined on the graphene layer by optical lithography and e-beam metal evaporation of $\mathrm{Ti}(10 \mathrm{~nm}) / \mathrm{Au}(150 \mathrm{~nm})$. Figure 2(d) displays an SEM image of the processed device with an open metal contact on the graphene layer. The close-up view of the metal stripe on the graphene layer (marked with a red rectangle in 

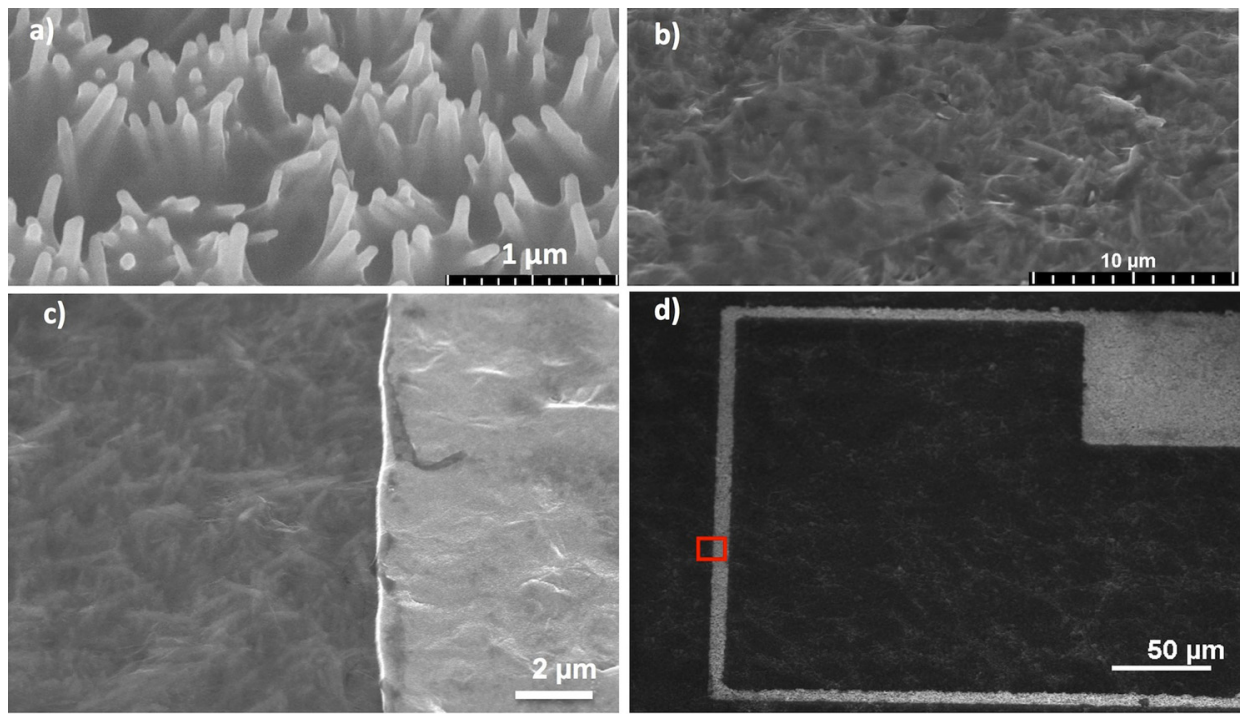

FIG. 2. $45^{\circ}$ tilted SEM images of (a) $\mathrm{ZnO}$ nanowires encapsulated into spinon glass; (b) graphene film transferred onto encapsulated nanowires; (c) and (d) processed device.
Fig. 2(d)) is shown in Figure 2(c)). The second contact is deposited on the nanowire surface outside the graphene layer as schematically shown in Fig. 3. The sample was mounted on a copper holder and wire-bonded for the electro-optical measurements.

The device operation principle is illustrated in Fig. 3. The detector is biased between two metal contacts that are deposited on the graphene layer and on bare $\mathrm{ZnO}$ nanowires, respectively. The excitation light is focused inside the open metal contact area, is transmitted by the transparent graphene sheet, and is absorbed in $\mathrm{ZnO}$ nanowire array under the graphene electrode. The photogenerated carriers are collected on one side by the top graphene contact with a metallization and on the other side are transported by the FTO layer and inactive $\mathrm{ZnO}$ nanowires under the second fully metallized contact.

It should be noted that the detector design can be further optimized by taking the second contact directly on the FTO layer and not on the $\mathrm{ZnO}$ nanowires. However, this would require an additional etching step to locally remove the $\mathrm{ZnO}$ nanowires together with encapsulating HSQ that we deliberately wanted to avoid.

\section{DEVICE CHARACTERIZATION}

Figure 4 compares the current-voltage (I-V) curves measured in the dark and under UV-light exposure at room temperature in ambient atmosphere. I-V data were recorded using a Keithley 2636 Source-Meter with $150 \mathrm{~ms}$ per point integration time. The illumination at $\lambda=357 \mathrm{~nm}$ was provided by a UV light emitting diode. The I-V curves in the dark show almost linear behavior characteristic of ohmic contacts. It should be noted that the work functions of $\mathrm{ZnO}$ $\left(4.25,4.64\right.$, and 4.95 depending on the face ${ }^{26}$ and of graphene (4.32-5.08 depending on the layer number and doping $^{27}$ ) are quite close, which is in favor of the ohmic contact formation. It should be noted that contrary to the present study, Schottky barrier between $\mathrm{ZnO}$ nanowires and the graphene layer has been reported in Ref. 12. This difference in the graphene/ $\mathrm{ZnO}$ contact properties should be due to the

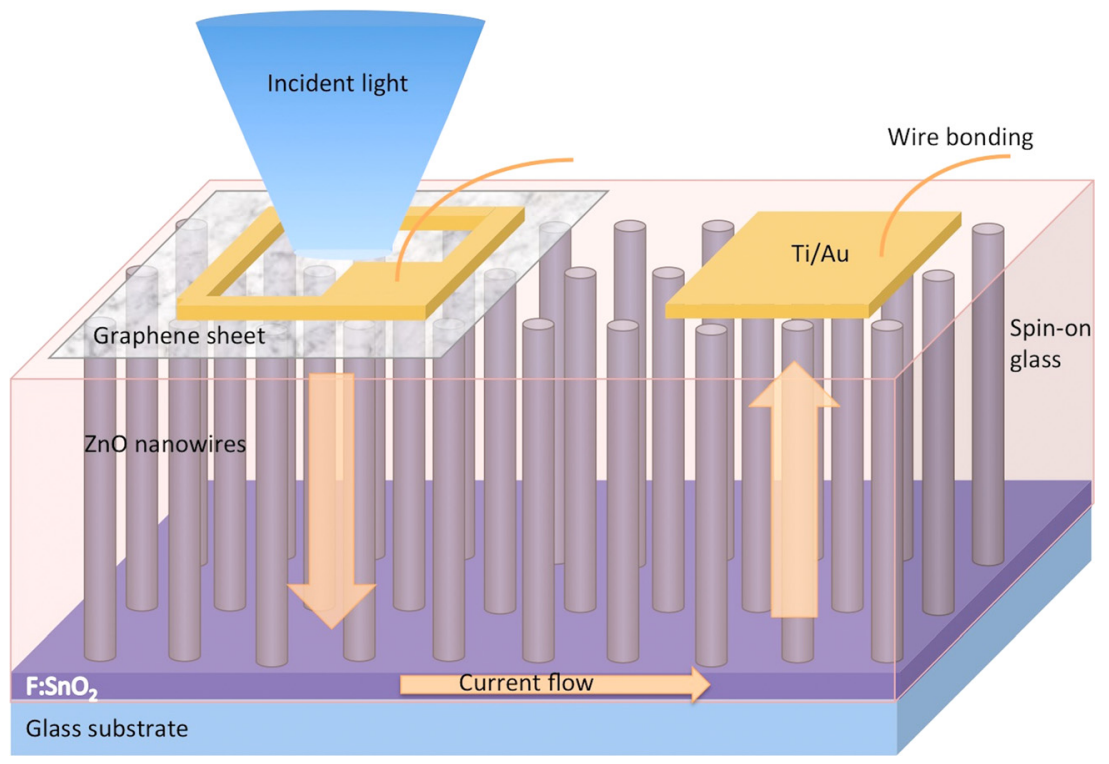

FIG. 3. Device schematic. 


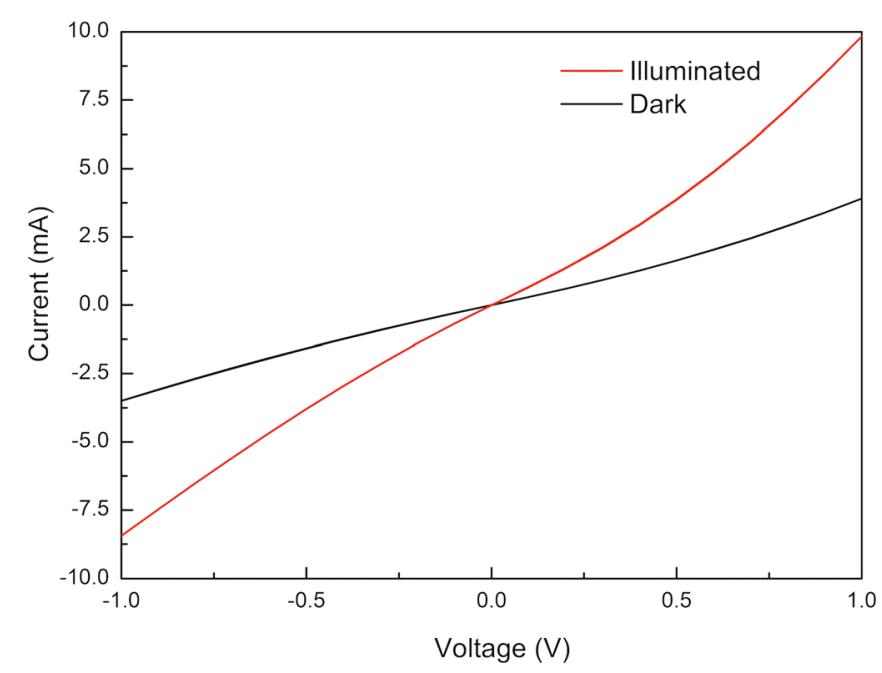

FIG. 4. I-V curves of the device in the dark and under UV illumination.

different work functions of the 4-ML graphene sheet used in this study and also to the higher residual n-type doping of $\mathrm{ZnO}$ nanowires grown by electrodeposition technique.

Under illumination with a power density of $8.5 \mathrm{~mW} / \mathrm{cm}^{2}$ (corresponding to a total power on the detector of $5.3 \mu \mathrm{W}$ ), the nanowire conductivity increases. The current steady state at $1 \mathrm{~V}$ is $3.9 \mathrm{~mA}(9.9 \mathrm{~mA})$ in the dark (under illumination).

To assess the spectral response of the detector, the photocurrent spectrum was measured at room temperature using a tunable visible-UV light source, consisting of a Xe lamp coupled with a Jobin Yvon Triax 180 spectrometer. The spectral resolution of the system used in this study is $\sim 40 \mathrm{meV}$. The spectrum is normalized by the optical system response. Figure 5 shows the PC spectrum measured under $20 \mathrm{mV}$ bias. The onset of the $\mathrm{PC}$ is at $3 \mathrm{eV}$, which is slightly below the PL peak energy $(3.25 \mathrm{eV})$ and the $\mathrm{ZnO}$ band gap $\left(3.3 \mathrm{eV}\right.$ at room temperature $\left.{ }^{28}\right)$. This sub-bandgap photocurrent can be attributed to a doping-related Urbach tail. It can

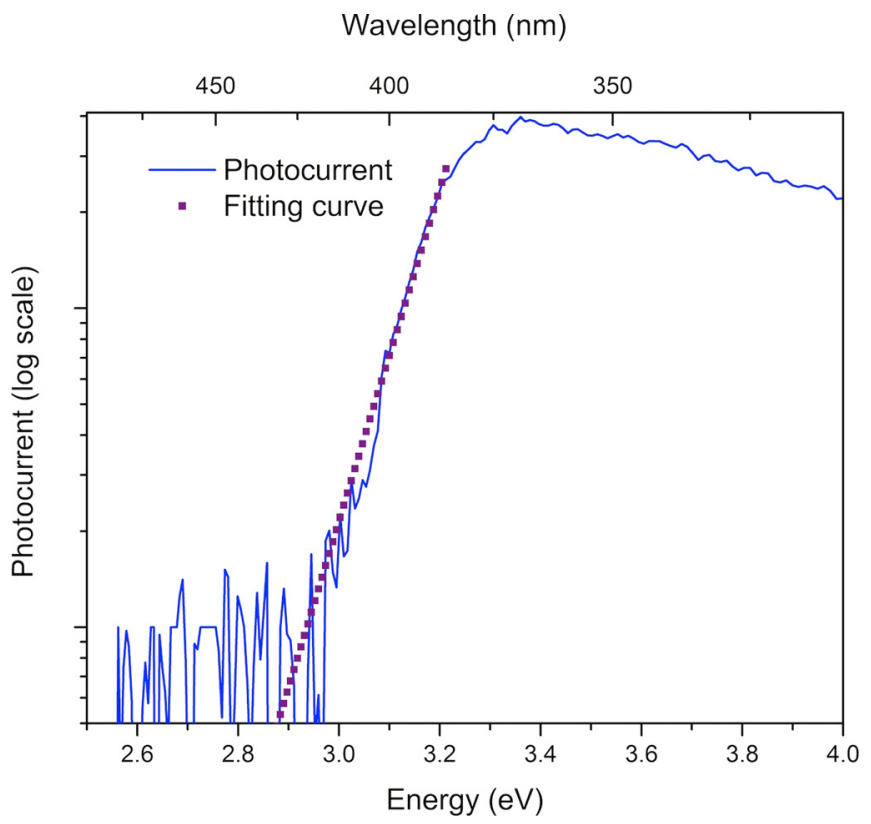

FIG. 5. Room temperature photocurrent spectrum with a fitting curve for the sub-bandgap part. be described with a characteristic energy $E_{U}$, which is related to the density of states in the gap. The photocurrent is expressed as $^{29}$

$$
I_{p h}=I_{0} \exp \left(\frac{E-E_{G}}{E_{U}}\right)
$$

where $I_{0}$ is the photocurrent value for the photon energy equal to the $\mathrm{ZnO}$ bandgap $E_{G}$.

Taking $E_{G}$ and $E_{U}$ as fitting parameters, the best fit of the photocurrent spectrum (Fig. 5) is obtained for $E_{G}=3.32 \mathrm{eV}$ and $E_{U}=83 \mathrm{meV}$. The Urbach energy is in reasonable agreement with the value of $76 \mathrm{meV}$ extracted from the absorption spectra of undoped $\mathrm{ZnO}$ thin films. ${ }^{30}$ For energies lower than $3.0 \mathrm{eV}$, the photocurrent is below the noise level.

The photocurrent increases rapidly with energy up to $3.365 \mathrm{eV}$ and then slowly decreases. This decrease can be attributed first to the reduction of the penetration depth of the light at shorter wavelengths so that only the small top portion of the nanowires is excited. A residual absorption of the spin-on glass encapsulation matrix may also participate to this signal reduction.

Figure 6 presents the time dependent current of the detector at a bias of $1 \mathrm{~V}$ for on/off illumination cycles $\left(\lambda=357 \mathrm{~nm}\right.$, power density $\left.8.5 \mathrm{~mW} / \mathrm{cm}^{2}\right)$. The current transient is recorded for an illumination time of $450 \mathrm{~s}$ and the current decay is followed during $600 \mathrm{~s}$. In the inset, the multiple on/off cycling is shown using a shorter illumination time of $5 \mathrm{~s}$ and an off time of $240 \mathrm{~s}$. The bi-exponential fitting of the time traces is also shown. First, the detector current increases rapidly under illumination from the initial value of $3.9 \mathrm{~mA}$ with an initial slope of $\sim 0.24 \mathrm{~mA} / \mathrm{s}$ (it should be noted that the temporal resolution of the used set-up is limited to response times larger than $0.5 \mathrm{~s}$ ). The current reaches $5.1 \mathrm{~mA}$ after $5 \mathrm{~s}$ and then the increase slows down. After $450 \mathrm{~s}$, the detector reaches the current of $9.5 \mathrm{~mA}$, which is still inferior to the steady state current $(9.9 \mathrm{~mA})$. The photocurrent builds up following a bi-exponential dependence

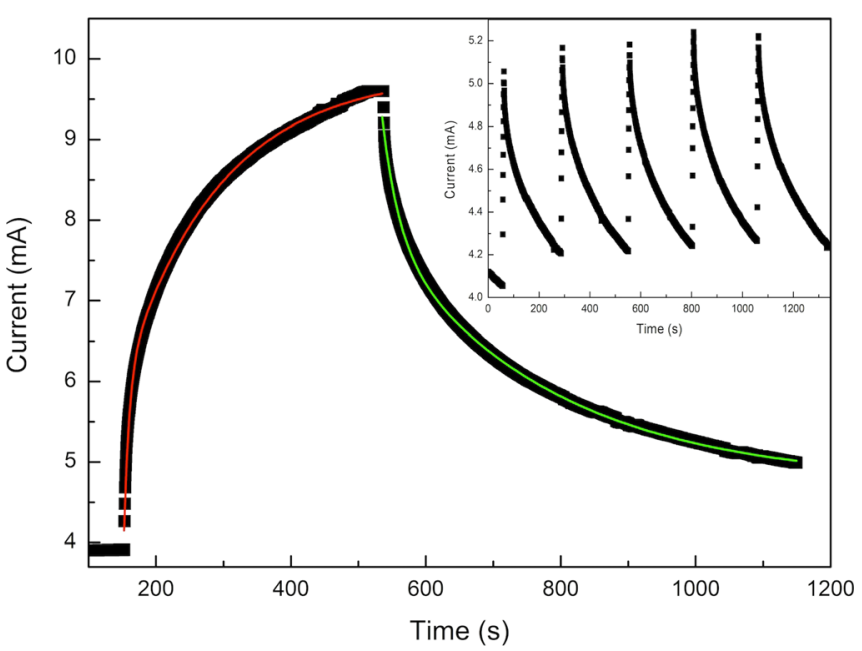

FIG. 6. Current transient under UV illumination during $450 \mathrm{~s}$ and relaxation during $600 \mathrm{~s}$. Inset shows the response to multiple illumination cycles with $5 \mathrm{~s}$ on and 240 off time. 
with time constants $t_{1}=7.7 \mathrm{~s}$ and $t_{2}=142 \mathrm{~s}$. The return to equilibrium is slower. The relaxation upon UV light off also exhibits a bi-exponential decay with significantly longer characteristic times $t_{1}=25 \mathrm{~s}$ and $t_{2}=254 \mathrm{~s}$. This complex multi-exponential dependence shows that the device photoresponse is governed by a large number of processes with different characteristic times. It should be due to the charge exchange with the environment such as $\mathrm{O}_{2}{ }^{-}$molecules neutralization and desorption at the top part of the free lateral surface or exchange of charged species with the encapsulating HSQ material. It is important to note that the current build-up time constant is smaller than the relaxation time constant showing that the holes trapping at the surface is a faster process then the release.

The power dependence of the photocurrent has been probed by illuminating the detector with a light emitting diode at $\lambda=357 \mathrm{~nm}$. The incident power has been varied by almost 4 orders of magnitude (the explored power density range is $7.2 \times 10^{-6}-2.2 \times 10^{-2} \mathrm{~W} / \mathrm{cm}^{2}$ ). The detector was biased at $1 \mathrm{~V}$. Figure 7 shows the photocurrent variation with the incident power density. At low excitation (power density below $2 \times 10^{-4} \mathrm{~W} / \mathrm{cm}^{2}$ ), the photocurrent scales linearly with power. Then, the dependence becomes sub-linear and the photocurrent almost saturates for power densities above $0.01 \mathrm{~W} / \mathrm{cm}^{2}$.

The responsivity $R$ of the detector defined as a ratio of the steady state photocurrent $I_{p h}=I_{\text {light }}-I_{\text {dark }}$ to the incident optical power $P_{\text {opt }}$

$$
R=\frac{I_{p h}}{P_{o p t}}
$$

is also reported in Figure 7. The responsivity is almost constant at low excitation reaching $1.4 \times 10^{4} \mathrm{~A} / \mathrm{W}$ for incident power density of $6 \times 10^{-5} \mathrm{~W} / \mathrm{cm}^{2}$. The corresponding photoconductive gain of the detector defined as a number of detected carriers per absorbed photon ${ }^{10,31}$

$$
G=\frac{N_{e}}{N_{p h}}=\frac{I_{p h}}{P_{o p t}} \times \frac{h v}{e}
$$

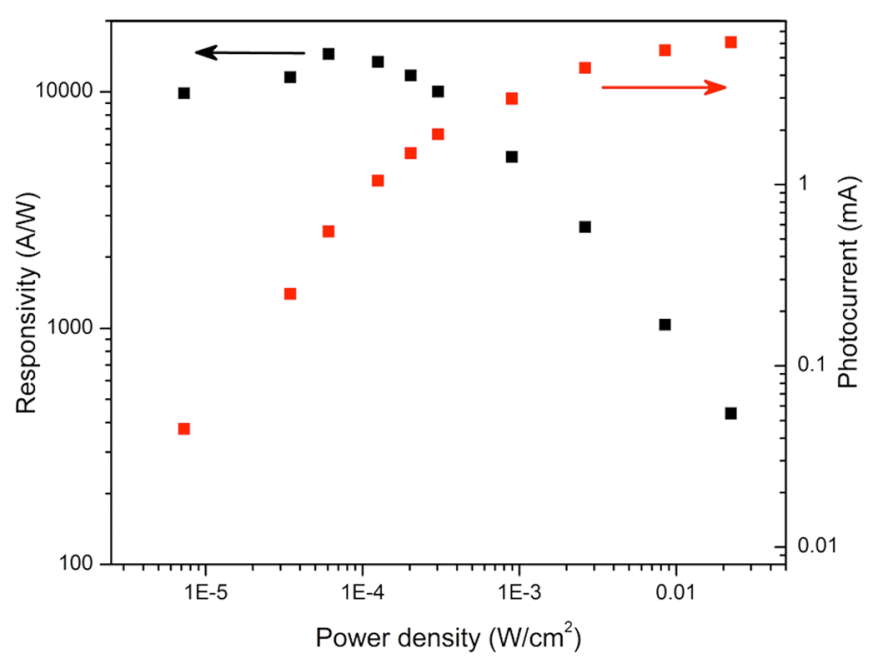

FIG. 7. Dependence of the photocurrent on the incident power density. is $5 \times 10^{4}$ assuming that the incident light is fully absorbed by the nanowires and neglecting the reflection losses. At high incident power, the responsivity decreases by more than one order of magnitude. This behavior is related to the photoconduction mechanism of the nanowire as will be discussed in Sec. V and can be explained by the flattening of the band bending and narrowing of the surface depletion layer at high light intensity.

\section{MODELING OF THE PHOTOCONDUCTIVE GAIN IN THE ZnO NANOWIRE PHOTODETECTOR}

For a homogeneous not fully depleted $\mathrm{ZnO}$ nanowire with ohmic contacts under a bias $V$, the current is given by the following expression:

$$
I=e \operatorname{Sn} \mu \frac{V}{L},
$$

where $\mu$ is the electron mobility, $e$ is the electron charge, $n$ is the volumic free electron density, $L$ is the nanowire length, and $S$ is the non-depleted cross-section surface of the nanowire.

Under illumination, an additional photocurrent is generated, which can be expressed as

$$
I_{p h}=\frac{e \mu V}{L}(S \Delta n+n \Delta S),
$$

where $\Delta n$ is the increase of the carrier density under illumination and $\Delta S$ is the variation of the conducting surface due to the modification of the band profile under illumination. Indeed, the Fermi level pinning at surface states creates a depleted region close to the lateral nanowire surface as illustrated in Figure 8. The concentration of the photogenerated carriers $\Delta n$ is proportional to the absorbed flux $\Delta n L=\phi \tau_{n}$, where $\phi$ is the number of photons absorbed in the nanowire per unit time and per unit surface and $\tau_{n}$ is the carrier recombination time. (It is noteworthy that at high excitation $\tau_{n}$ depends on the incident flux as discussed in Ref. 10.)

The photoconductive gain of the detector can be expressed as $G=\frac{N_{e}}{N_{p h}}=\frac{I_{p h} / e}{\phi S}$. We define the carrier transit time $\tau_{t}=\frac{L}{v}=\frac{L^{2}}{\mu v}$, where $v$ is the electron velocity. Then, the expression for the photoconductive gain takes the form

$$
G=\frac{\tau_{n}}{\tau_{t}}+\frac{n L \Delta S}{\tau_{t} \phi S} .
$$

The first term is the habitual expression for the gain, which is the ratio of carrier lifetime to carrier transit time. It can be understood as a number of times, the photogenerated carrier goes around the electrical circuit before recombination. The transit time for a $1 \mu \mathrm{m}$ long $\mathrm{ZnO}$ nanowire under $1 \mathrm{~V}$ bias taking an electron mobility of $1000 \mathrm{~cm}^{2} / \mathrm{Vs}^{32}$ is about $10 \mathrm{ps}$. [In the literature, there is a large dispersion of mobility values for $\mathrm{ZnO}$ nanowires, e.g., $13 \pm 5 \mathrm{~cm}^{2} / \mathrm{Vs},{ }^{35} 17 \mathrm{~cm}^{2} / \mathrm{Vs},{ }^{36}$ $75 \mathrm{~cm}^{2} /$ Vs for bare nanowires, and $1000 \mathrm{~cm}^{2} /$ Vs for coated nanowires. ${ }^{32}$ To get the upper estimate of the gain, the highest value was chosen.] The carrier lifetime can be estimated from the time-resolved photoluminescence measurements 


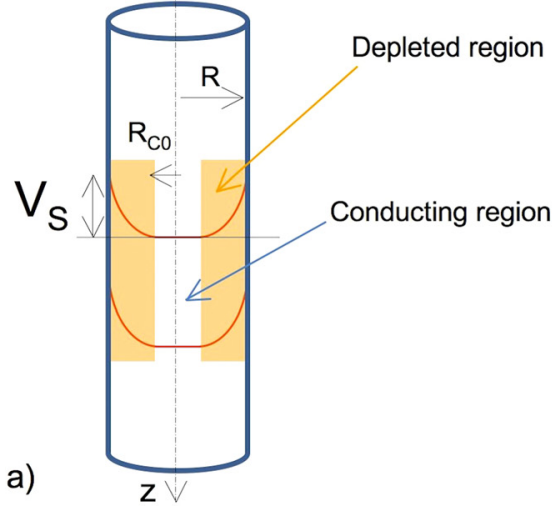

b)

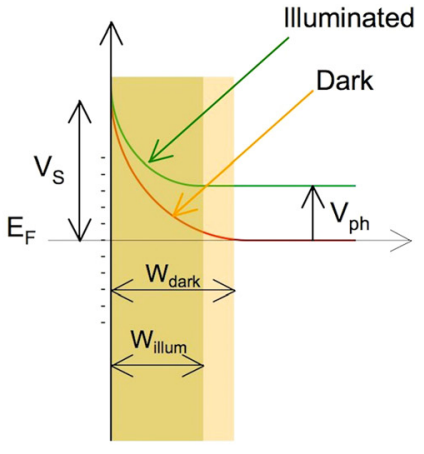

FIG. 8. (a)-Schematic illustrating the conduction and valence bands bending at the nanowire surface in the dark. (b)-Modification of the conduction band profile along the nanowire radius in the dark and under illumination. giving values of $350 \mathrm{ps}^{2}$ Therefore, the first term of the gain expression can account for gain values of the order of 30 , which does not allow to explain the observed high gain.

The second term in (3) reflects the modulation of the conducting cross-section due to the hole trapping at the lateral surface similar to the model described by Garrido et al $^{33}$ for $2 \mathrm{D}$ case. However, for the specific nanowire geometry with a very high surface to volume ratio, this term can be much stronger than in the $2 \mathrm{D}$ case.

In order to calculate the variation of the conducting surface $\Delta S$ due to the modulation of the depletion region, we calculate the lateral band profile for a nanowire in the dark and under illumination at each point $z$. We approximate the nanowire as a cylinder of a radius $R$ as illustrated in Fig. 8 . The $z$-axis is directed towards the substrate with $z=0$ corresponding to the nanowire top. Since the light is exponentially attenuated as it propagates from the top surface towards the substrate in the ensemble of vertically standing nanowires, the conducting area under illumination depends on $z$. Therefore, we will consider a nanowire segment $[z, z+\Delta z]$, for which the photon flux is constant.

Following, ${ }^{34}$ the potential profile $V$ in the dark can be found by solving the Poisson equation in cylindrical coordinates:

$$
\frac{\partial^{2} V}{\partial r^{2}}+\frac{1}{r} \frac{\partial V}{\partial r}=-\frac{e N_{d}}{\varepsilon \varepsilon_{0}}
$$

where $N_{d}$ is the nanowire volumic doping (all donors are supposed ionized). We note $R_{C O}$ the radius of the nondepleted conducting surface in the dark: $S=\pi R_{C 0}^{2}$ as illustrated in Fig. 8 and suppose that both the built-in potential and the electric field are zero at $r=R_{C 0}$. The solution in the depleted region $R_{C 0}<r<R$ is given by

$$
V(r)=\frac{e N_{d} R_{C 0}^{2}}{2 \varepsilon \varepsilon_{0}}\left(\ln \left(\frac{r}{R_{C 0}}\right)-\frac{r^{2}}{2 R_{C 0}^{2}}+\frac{1}{2}\right) .
$$

At the nanowire surface at $r=R$, the potential is pinned by surface states at a value $V_{S}$, which we consider as a parameter known from experiment

$$
V_{S}=\frac{e N_{d} R_{C 0}^{2}}{2 \varepsilon \varepsilon_{0}}\left(\ln \left(\frac{R}{R_{C 0}}\right)-\frac{R^{2}}{2 R_{C 0}^{2}}+\frac{1}{2}\right) .
$$

Contrary to the planar case ${ }^{33}$ the dependence of the radius of conducting area in the dark $R_{C 0}$ on the surface potential $V_{S}$ cannot be obtained analytically. However, the numerical solution $R_{C 0}\left(V_{S}\right)$ of Eq. (4) can be easily found.

Using expression (2), the dark current of the nanowire can be calculated as a function of $R_{C 0}$

$$
I_{\text {dark }}=\frac{\pi R_{C 0}^{2}}{L} e \mu n V .
$$

Under illumination, the excess of holes is attracted to the surface and is trapped at the surface states. This phenomenon modifies the potential profile and reduces the depletion region. To calculate the modification of the built-in potential at the nanowire center $V_{p h}$, we consider the new equilibrium state of the nanowire under illumination. At equilibrium, the thermionic current of carriers released from the surface $I_{T E}^{S \rightarrow}$ should compensate the thermionic current of carriers trapped at the surface $I_{T E}^{S \leftarrow}$ plus the lateral photocurrent generated by the illumination in the space charge region $I_{p h}^{\text {lat }}$

$$
I_{T E}^{S \rightarrow}-I_{T E}^{S \leftarrow}-I_{p h}^{l a t}=0
$$

By analogy with a Schottky diode, the thermionic currents can be expressed as

$$
\begin{gathered}
I_{T E}^{S \leftarrow}=2 \pi R \Delta z A^{*} T^{2} \exp \left(\frac{V_{p h}-V_{S}}{\frac{k_{B} T}{e}}\right), \\
I_{T E}^{S \rightarrow}=2 \pi R \Delta z A^{*} T^{2} \exp \left(\frac{V_{p h}}{\frac{k_{B} T}{e}}\right),
\end{gathered}
$$

where $A^{*}$ is the Richardson constant, $\mathrm{T}$ is the temperature, and $k_{B}$ is Boltzmann constant.

Considering that the lateral photocurrent $I_{p h}^{l a t}$ is generated only in the depleted region, it can be expressed as

$$
I_{p h}^{l a t}=e \Delta \phi \pi\left(R^{2}-R_{C}^{2}\right)
$$

where $\Delta \phi$ is the photon flux absorbed in the nanowire segment $[z, z+\Delta z], R_{C}$ is the radius of the conducting area under illumination.

Equation (6) can be rewritten as 


$$
V_{p h}=\frac{k_{B} T}{e} \ln \left(1+e \frac{R^{2}-R_{C}^{2}}{2 R A^{*} T^{2}} \frac{d \phi}{d z} \exp \left(\frac{V_{S}}{\frac{k_{B} T}{e}}\right)\right)
$$

As an approximation, the optical power density can be described as $P=P_{0} \exp \left(-\alpha_{e f f} z\right)$, where $P_{O}$ is the incident power density and $\alpha_{e f f}$ is the effective absorption coefficient of the nanowire ensemble (reflection losses are neglected). Using the relation between the optical power density and the photon flux $\phi=\frac{P}{h \nu}$, where $h \nu$ is the photon energy, the expression for $V_{p h}$ becomes

$V_{p h}=\frac{k_{B} T}{e} \ln \left(1-\frac{e}{h v} \frac{R^{2}-R_{C}^{2}}{2 R A^{*} T^{2}} \alpha_{e f f} P_{0} \exp \left(-\alpha_{e f f} z\right) \exp \left(\frac{V_{S}}{\frac{k_{B} T}{e}}\right)\right)$.

Coming back to Eq. (4), we can rewrite it for a nanowire under illumination, if we replace $V_{S}$ by $V_{S}-V_{p h}$, and $R_{C 0}$ by $R_{C}$

$$
V_{S}-V_{p h}=\frac{e N_{d} R_{C}^{2}}{2 \varepsilon \varepsilon_{0}}\left(\ln \left(\frac{R}{R_{C}}\right)-\frac{R^{2}}{2 R_{C}^{2}}+\frac{1}{2}\right)
$$

The radius of the conducting area under illumination $R_{C}(z)$ can now be found by solving self-consistently Eqs. (7) and (8).

The expression (2) can be rewritten in order to relate the current under illumination with applied bias and the integrated resistance of the nanowire as

$$
\begin{aligned}
V & =I_{\text {light }} \frac{1}{e \mu} \int_{0}^{L} \frac{d z}{S(z) n(z)} \\
& =I_{\text {light }} \frac{1}{e \mu} \int_{0}^{L} \frac{d z}{S_{\text {dark }} n_{\text {dark }}+S_{\text {dark }} \Delta n(z)+n_{\text {dark }} \Delta S(z)},
\end{aligned}
$$

where the conducting surface $S(z)=S_{\text {dark }}+\Delta S(z)$ $=\pi R_{C}^{2}(z)$. We assume that $\frac{\Delta n(z)}{n_{\text {dark }}} \ll 1$, which is a reasonable assumption taking into account the high residual doping of the $\mathrm{ZnO}$ nanowires grown by electrodeposition method. In this approximation, the current under illumination can be expressed as

$$
I_{\text {light }}=\frac{\pi e \mu n V}{\int_{0}^{L} \frac{d z}{R_{C}^{2}(z)}}
$$

The photocurrent is found by subtracting the expressions (9) and (5) and the photoconductive gain is calculated using definition (1).

Under a simplifying hypothesis that all nanowires are identical, the gain of the nanowire ensemble equals that of a single nanowire.

The calculated photoconductive gain is shown in Figure 9 for different nanowire diameters. In this calculation, we have used the following parameters: a nanowire doping of $2 \times 10^{18} \mathrm{~cm}^{-3}$, a nanowire length $1 \mu \mathrm{m}$, a value of Richardson constant of $32 \mathrm{Acm}^{2} \mathrm{~K}^{-2}, 37$ a temperature

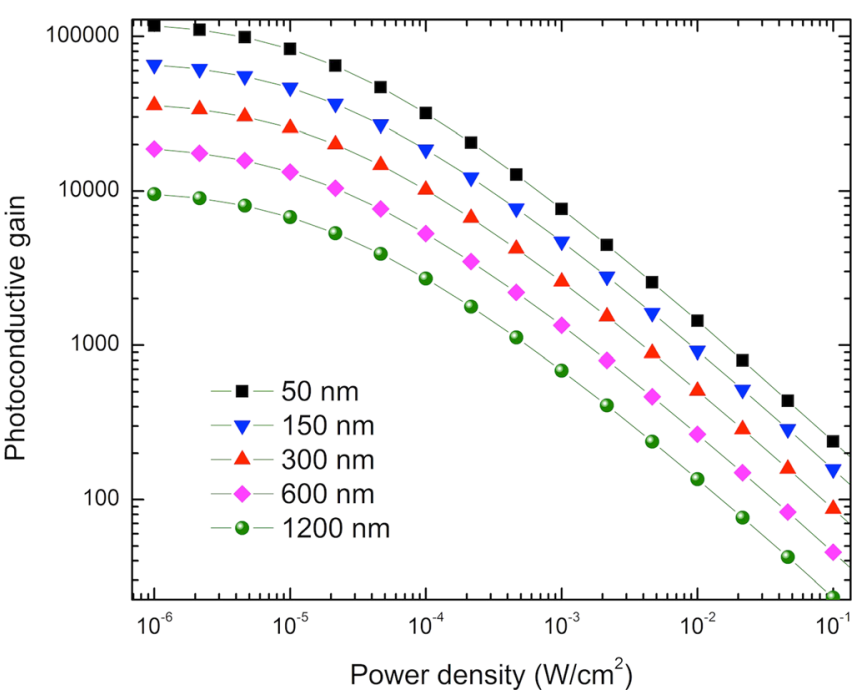

FIG. 9. Calculated dependence of the photoconductive gain on the power density for different nanowire diameters.

$\mathrm{T}=300 \mathrm{~K}$, an absorption coefficient $\alpha_{\text {eff }}=2 \times 10^{5} \mathrm{~cm}^{-1},{ }^{38} \mathrm{a}$ nanowire conductivity $\sigma=e \mu n=0.013 \mathrm{~S} / \mathrm{cm}$, and a surface potential $V_{d}=0.29 \mathrm{eV}$. This last parameter is not well known in the literature. Indeed, the band bending is caused by the total surface charge, which is a combination of surface charge due to surface states within the gap and surface-fixed charge that are not related to the surface traps (e.g., adsorbed charged species like oxygen). Therefore, the surface potential is influenced by the growth conditions, the surface treatment and even the environment, which means that it is sample dependent. The chosen value of $V_{d}$ corresponds to the best fit with the experimental data.

By using the gain expression derived for a single nanowire to describe the nanowire ensemble, we assume that all nanowires are electrically contacted. However, the height inhomogeneities may reduce the effective number of contacted nanowires. This would in consequence reduce the photoconductive gain by a factor $\mathrm{N}^{\text {contacted }} / \mathrm{N}^{\text {illuminated }}$, where $\mathrm{N}^{\text {contacted }}$ is the number of contacted nanowires and the $\mathrm{N}^{\text {illuminated }}$ is the total number of illuminated nanowires. This parameter is difficult to quantify because of the complexity of nanowire ensemble morphology. However, it can be incorporated in the nanowire conductivity by lowering its value to account for electrically inactive nanowires. The above-mentioned nanowire conductivity $\sigma=0.013 \mathrm{~S} / \mathrm{cm}$ was chosen to produce the best fit with the experimental data.

At low excitation power, the model predicts a photoconductive gain up to $10^{5}$ for nanowire radius of $50 \mathrm{~nm}$. The gain decreases when increasing the nanowire diameter. Indeed, as the conducting surface increases, the variation of the surface depletion layer has a weaker effect on the nanowire conductivity. The power dependence is qualitatively similar for the different diameters. The gain varies slowly at power densities below $10^{-5} \mathrm{~W} / \mathrm{cm}^{2}$ and then the slope becomes steeper. The reduction of the gain at high excitation power originates from the shrinking of the depletion region. In this case, the conducting surface approaches the maximum possible surface of the nanowire cross-section and the $\Delta \mathrm{S}$ modulation becomes negligible. 


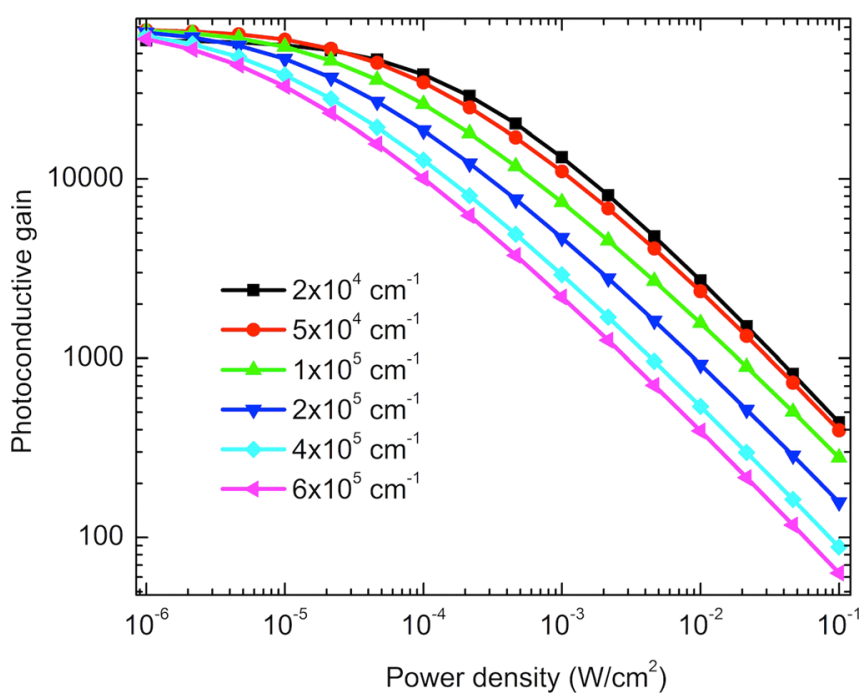

FIG. 10. Calculated dependence of the photoconductive gain on the power density for different values of the effective absorption coefficient $\alpha_{e f f}$.

Figure 10 illustrates the influence of the effective absorption coefficient on the gain. We use the same parameters as before and the nanowire diameter is $150 \mathrm{~nm}$. The dense nanowire ensemble is considered as an effective medium described by an effective absorption coefficient. To explore the influence of the absorption coefficient on the gain, the value of $\alpha_{e f f}$ was varied from $2 \times 10^{4}$ to $6 \times 10^{5} \mathrm{~cm}^{-1}$. As seen in Fig. 10, the photoconductive gain is reduced when the absorption coefficient increases. The dependence at low excitation power becomes steeper. The origin of the gain reduction is the decrease of the penetration depth for higher absorption coefficient, so that the conducting surface variation takes place in a shorter portion of the nanowire close to the sample surface. This result also explains the spectral dependence of the photocurrent (Fig. 5) showing a photocurrent decrease at high energies. Indeed, the $\mathrm{ZnO}$ absorption coefficient increases above the bandgap $^{38}$ leading to the gain reduction at shorter wavelengths.

The above-described model was used to fit the experimental results. Figure 11 displays the comparison of the

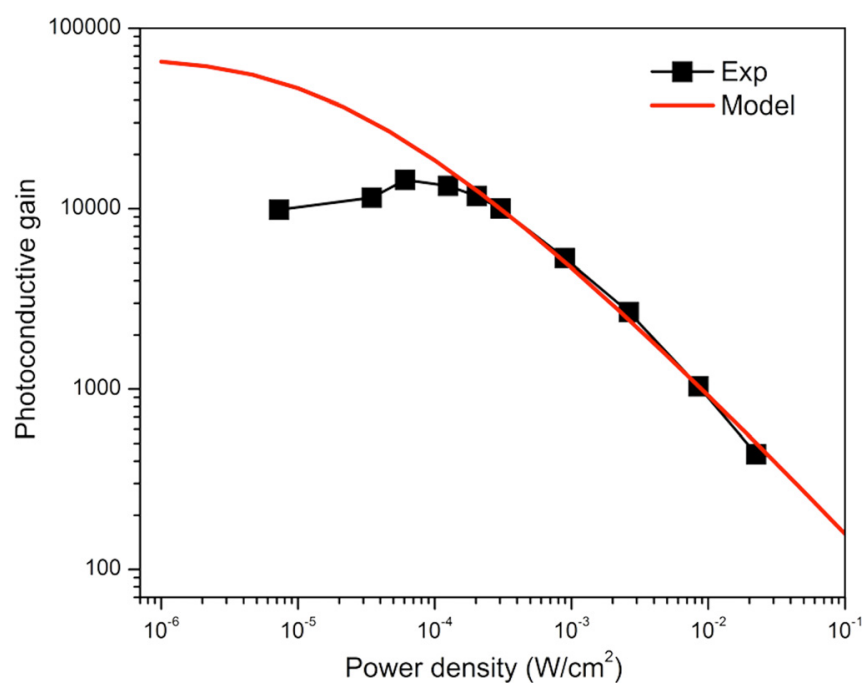

FIG. 11. Comparison of the calculated (red line) and measured (black squares) values of the photoconductive gain. measured gain power dependence with simulations using the same parameter values same as used in Fig. 9 and the nanowire diameter of $150 \mathrm{~nm}$. An excellent quantitative agreement is obtained for an excitation power density above $10^{-4}$ $\mathrm{W} / \mathrm{cm}^{2}$. The slope is well reproduced over more than 2 orders of magnitude of power densities. At low excitation power, simulations overestimate the gain by a factor of 6 with respect to the experimental values. The lower measured gain values may be due to some carrier loss at the surface, which has been neglected in the calculation of the built-in potential profile. Despite this small deviation, the model correctly predicts the gain power dependence and confirms that the variation of the conducting surface is the main mechanism responsible for the photoconductive gain.

\section{CONCLUSION}

In summary, we have demonstrated that graphene can be successfully used as a transparent electrode for photodetectors based on $\mathrm{ZnO}$ nanowire ensembles. The fabricated device demonstrates high responsivity (above $10^{4} \mathrm{~A} / \mathrm{W}$ ) in the near UV spectral range. Photocurrent spectroscopy reveals a weak sub-bandgap response down to $415 \mathrm{~nm}$, which can be described by an Urbach tail with a characteristic energy of $83 \mathrm{meV}$. The detector responsivity is degraded at high excitation power. A comprehensive model of the photoconductive mechanism is proposed showing that the main process responsible for the photoconductive gain is the modulation of the conducting surface due to the variation of the surface depletion layer. The model predicts a gain decrease at high power in agreement with the experimental data.

\section{ACKNOWLEDGMENTS}

The authors acknowledge the financial support from the FP7 EU Project "Funprob" (Grant Agreement PIRSES-GA2010-269169), from the C-Nano IdF Project "EffiNanoLED," as well as from the Russian Foundation for Basic Research and Grant of St. Petersburg Government.

${ }^{1}$ M. Razeghi and A. Rogalski, "Semiconductor ultraviolet detectors," J. Appl. Phys. 79(10), 7433-7473 (1996).

${ }^{2}$ M. H. Huang, S. Mao, H. Feick, H. Yan, Y. Wu, H. Kind, E. Weber, R. Russo, and P. Yang, "Room-temperature ultraviolet nanowire nanolasers," Science 292(5523), 1897-1899 (2001).

${ }^{3} \mathrm{~S}$. Basu and A. Dutta, "Modified heterojunction based on zinc oxide thin film for hydrogen gas-sensor application," Sens. Actuators B 22(2), 83-87 (1994).

${ }^{4}$ Q. Wan, Q. H. Li, Y. J. Chen, T. H. Wang, X. L. He, J. P. Li, and C. L. $\mathrm{Lin}$, "Fabrication and ethanol sensing characteristics of $\mathrm{ZnO}$ nanowire gas sensors," Appl. Phys. Lett. 84(18), 3654-3656 (2004).

${ }^{5}$ K. Liu, M. Sakurai, and M. Aono, "ZnO-based ultraviolet photodetectors," Sensors 10(9), 8604-8634 (2010).

${ }^{6} \mathrm{H}$. Kind, H. Yan, B. Messer, M. Law, and P. Yang, "Nanowire ultraviolet photodetectors and optical switches," Adv. Mater. 14(2), 158 (2002).

${ }^{7}$ O. Lupan, V. M. Guérin, I. M. Tiginyanu, V. V. Ursaki, L. Chow, H. Heinrich, and T. Pauporté, "Well-aligned arrays of vertically oriented $\mathrm{ZnO}$ nanowires electrodeposited on ITO-coated glass and their integration in dye sensitized solar cells," J. Photochem. Photobiol., A 211(1), 65-73 (2010).

${ }^{8}$ O. Lupan and T. Pauporté, "Hydrothermal treatment for the marked structural and optical quality improvement of $\mathrm{ZnO}$ nanowire arrays deposited 
on lightweight flexible substrates," J. Cryst. Growth 312(16), 2454-2458 (2010).

${ }^{9}$ M. E. Swanwick, S. M. Pfaendler, A. I. Akinwande, and A. J. Flewitt, "Near-ultraviolet zinc oxide nanowire sensor using low temperature hydrothermal growth," Nanotechnology 23(34), 344009 (2012).

${ }^{10}$ C. Soci, A. Zhang, B. Xiang, S. A. Dayeh, D. P. R. Aplin, J. Park, X. Y. Bao, Y. H. Lo, and D. Wang, "ZnO nanowire UV photodetectors with high internal gain," Nano Lett. 7(4), 1003-1009 (2007).

${ }^{11}$ C.-H. Chen and C. T. Lee, "High detectivity mechanism of ZnO-based nanorod ultraviolet photodetectors," IEEE Photon. Technol. Lett. 25(4), 348-351 (2013).

${ }^{12}$ X. W. Fu, Z. M. Liao, Y. B. Zhou, H. C. Wu, Y. Q. Bie, J. Xu, and D. P. Yu, "Graphene/ZnO nanowire/graphene vertical structure based fast-response ultraviolet photodetector," Appl. Phys. Lett. 100(22), 223114 (2012).

${ }^{13}$ E. S. Ates, S. Kucukyildiz, and H. E. Unalan, "Zinc oxide nanowire photodetectors with single-walled carbon nanotube thin-film electrodes," ACS Appl. Mater. Interfaces 4(10), 5142-5146 (2012).

${ }^{14}$ H. X. Chang, Z. H. Sun, K. Y. Ho, X. M. Tao, F. Yan, W. M. Kwok, and Z. J. Zheng, "A highly sensitive ultraviolet sensor based on a facile in situ solution-grown $\mathrm{ZnO}$ nanorod/graphene heterostructure," Nanoscale 3(1), 258-264 (2011).

${ }^{15}$ J. Yi, J. M. Lee, and W. I. Park, "Vertically aligned ZnO nanorods and graphene hybrid architectures for high-sensitive flexible gas sensors," Sens. Actuators B 155(1), 264-269 (2011).

${ }^{16}$ B. Nie, J. G. Hu, L. B. Luo, C. Xie, L. H. Zeng, P. Lv, F. Z. Li, J. S. Jie, M. Feng, C. Y. Wu, Y. Q. Yu, and S. H. Yu, "Monolayer graphene film on $\mathrm{ZnO}$ nanorod array for high performance Schottky junction ultraviolet photodetectors," Small 9(17), 2872-2879 (2013).

${ }^{17}$ T. Pauporté, D. Lincot, B. Viana, and F. Pellé, "Toward laser emission of epitaxial nanorod arrays of $\mathrm{ZnO}$ grown by electrodeposition," Appl. Phys. Lett. 89(23), 233112 (2006)

${ }^{18}$ H. E. Belghiti, T. Pauporté, and D. Lincot, "Mechanistic study of $\mathrm{ZnO}$ nanorod array electrodeposition," Phys. Status Solidi A 205(10), 2360-2364 (2008).

${ }^{19}$ O. Lupan, T. Pauporté, and B. Viana, "Low voltage UV electroluminescence from $\mathrm{ZnO}$ nanowire array/p GaN light emitting diodes,"Adv. Mater. 22(30), 3298-3302 (2010).

${ }^{20}$ O. Lupan, T. Pauporté, B. Viana, I. M. Tiginyanu, V. V. Ursaki, and R. Cortes, "Epitaxial electrodeposition of $\mathrm{ZnO}$ nanowire arrays on $\mathrm{p}-\mathrm{GaN}$ for efficient UV-light-emitting diode fabrication," ACS Appl. Mater. Interfaces 2(7), 2083-2090 (2010).

${ }^{21}$ T. Pauporté, G. Bataille, L. Joulaud, and F. J. Vermersch, "Well-aligned $\mathrm{ZnO}$ nanowire arrays prepared by seed-layer-free electrodeposition and their Cassie-Wenzel transition after hydrophobization,” J. Phys. Chem. C 114(1), 194-202 (2010).

${ }^{22}$ P. Mandin, T. Pauporté, P. Fanouillère, and D. Lincot, "Modelling and numerical simulation of hydrodynamical processes in a confined rotating electrode configuration,” J. Electroanal. Chem. 565(2), 159-173 (2004).
${ }^{23}$ T. Pauporté, E. Jouanno, F. Pellé, B. Viana, and P. Aschehoug, "Key growth parameters for the electrodeposition of $\mathrm{ZnO}$ films with an intense UV-light emission at room temperature," J. Phys. Chem. C 113(24), 10422-10431 (2009).

${ }^{24}$ A. V. Babichev, V. E. Gasumyants, and V. Y. Butko, "Resistivity and thermopower of graphene made by chemical vapor deposition technique," J. Appl. Phys. 113(7), 076101 (2013).

${ }^{25}$ A. V. Babichev, V. Y. Butko, M. S. Sobolev, E. V. Nikitina, N. V. Kryzhanovskaya, and A. Y. Egorov, "Electroluminescence of $\mathrm{GaP}_{\mathrm{x}} \mathrm{N}_{\mathrm{y}} \mathrm{As}_{1-\mathrm{x}-\mathrm{y}}$ nanoheterostructures through a transparent electrode made of CVD graphene," Semiconductors 46(6), 796-800 (2012).

${ }^{26} \mathrm{H}$. Moormann, D. Kohl, and G. Heiland, "Work function and band bending on clean cleaved zinc oxide surfaces," Surf. Sci. 80, 261-264 (1979).

${ }^{27}$ T. H. Han, Y. Lee, M. R. Choi, S. H. Woo, S. H. Bae, B. H. Hong, J. H. Ahn, and T. W. Lee, "Extremely efficient flexible organic light-emitting diodes with modified graphene anode," Nature Photon. 6(2), 105-110 (2012).

${ }^{28}$ V. Srikant and D. R. Clarke, "On the optical band gap of zinc oxide," J. Appl. Phys. 83, 5447 (1998).

${ }^{29} \mathrm{~F}$. Urbach, "The long-wavelength edge of photographic sensitivity and of the electronic absorption of solids," Phys. Rev. 92, 1324 (1953).

${ }^{30}$ R. C. Rai, "Analysis of the Urbach tails in absorption spectra of undoped ZnO thin films," J. Appl. Phys. 113(15), 153508 (2013)

${ }^{31}$ R. H. Bube, Photoconductivity of Solids (Wiley, New York, 1960), p. 60.

${ }^{32}$ W. I. Park, J. S. Kim, G. C. Yi, M. H. Bae, and H. J. Lee, "Fabrication and electrical characteristics of high-performance $\mathrm{ZnO}$ nanorod field-effect transistors," Appl. Phys. Lett. 85(21), 5052-5054 (2004).

${ }^{33}$ J. A. Garrido, E. Monroy, I. Izpura, and E. Munoz, "Photoconductive gain modelling of GaN photodetectors," Semicond. Sci. Technol. 13(6), 563 (1998).

${ }^{34}$ V. Dobrokhotov, D. N. McIlroy, M. G. Norton, A. Abuzir, W. J. Yeh, I. Stevenson, R. Pouy, J. Bochenek, M. Cartwright, L. Wang, J. Dawson, M. Beaux, and C. Berven, "Principles and mechanisms of gas sensing by GaN nanowires functionalized with gold nanoparticles," J. Appl. Phys. 99(10), 104302 (2006).

${ }^{35}$ H. Chen, J. Wang, H. Yu, H. Yang, S. Xie, and J. Li, “Transmission electron microscopy study of pseudoperiodically twinned $\mathrm{Zn}_{2} \mathrm{SnO}_{4}$ nanowires," J. Phys. Chem. B 109(7), 2573-2577 (2005).

${ }^{36} \mathrm{Z}$. Fan, D. Wang, P. C. Chang, W. Y. Tseng, and J. G. Lu, "ZnO nanowire field-effect transistor and oxygen sensing property,” Appl. Phys. Lett. 85, $5923(2004)$

${ }^{37}$ T. J. Hsueh, C. L. Hsu, S. J. Chang, Y. R. Lin, T. S. Lin, and I. C. Chen, "Growth and characterization of sparsely dispersed $\mathrm{ZnO}$ nanowires," J. Electrochem. Soc. 154(3), H153-H156 (2007).

${ }^{38}$ J. F. Muth, R. M. Kolbas, A. K. Sharma, S. Oktyabrsky, and J. Narayan, "Excitonic structure and absorption coefficient measurements of $\mathrm{ZnO}$ single crystal epitaxial films deposited by pulsed laser deposition," J. Appl. Phys. 85(11), 7884-7887 (1999). 